\title{
'Tensile Strength Determination of the Interface Between Porcelain Fused to Gold
}

\author{
MIKE KELLY and KAMAL ASGAR, School of Dentistry, Univer- \\ sity of Michigan, Ann Arbor, Michigan 48104 and WILLIAM J. \\ O'BRIEN, School of Dentistry, Marquette University, Milwaukee, \\ Wisconsin 53233
}

\section{Synopsis}

A method of testing the tensile strength of the porcelain-gold bond has been developed. Tensile strengths of the bond are improved primarily by the formation of an oxide layer on the gold before baking the porcelain and not by the degree of gross surface roughness. The oxide layer may act either as a transition ayer or it may create microsurface roughness. The gross surface roughness probably complicates the wetting action of the porcelain on the gold. Whether the actual bond is of a chemical nature, physical nature, or both, remains uncertain.

The use of enamel-metal composite structures in dentistry is increasing rapidly. As a result, there has been considerable interest in the nature and the strength of the junction formed between porcelain baked onto metals.

During the last decade, many investigators have studied the bond strength between the cast structure and enamel baked onto it. Shell and Nielson, ${ }^{1}$ in their work with a pull-through shear-type test, have found the effect of different variables on the bond strength. According to their study, an addition of trace metals to gold alloys triples the strength of the bond. They also found that roughness of the metal surface does not influence the bond strength apprecialy. This test seems to be rather complex and involves compressive residual stresses, as well as forces of adhesion. Johnston et al., ${ }^{2}$ with an adhesive-bonding test that consists of two rods of cast gold held in axial alignment and joined by porcelain, obtained a mean value of 5620 psi. However, in each case the porcelain fractured in porcelain 
and not at the interface. The wide range of values were due to failure to keep the gold rods aligned during the firing. Silver et al., ${ }^{3}$ using a setup similar to the one used by Johnston, have recorded values in the range of 3000 psi using improved Ceramco gold. In discussing the nature of the bond, they claim that much of the force of adhesion resulted from physical entanglement of gold and porcelairixparticles during fusion. Naturally, there will also be some molecular adhesion due to electrostatic forces. They also postulated that there may be chemical adhesion resulting from the reaction between porcelain and the metallic oxides of the gold. However, this test is similar to a cantilever beam type, the results of which are difficult to assess. Custer, ${ }^{4}$ by means of a transverse test, showed similar results. Vickery ${ }^{5}$ claims the only true bonding effect derives from direct reaction of porcelain with interstitial compounds developed at grain boundaries in the metal substrate. No evidence has been found for a chemical bond between porcelain and gold per se.

Since the results of a tensile test of a junction are more easily interpreted, it was the purpose of this experiment to design a specimen to measure only the tensile strength of the bond. An effort was made to determine the influence of various surface condition and the influence of preoxidation of the metal. Finally, an attempt was made to decide on a possible mechanism for the bonding phenomena via data obtained by this work.

\section{Materials and Methods}

The two factors investigated in this study were the surface roughness and prior oxidation of the alloy surfaces. A simple factorial design was employed as illustrated in Table I. Eight replications were made per treatment.

Rods of a commercial gold alloy (Ceramco No. 1, Ceramco Inc., Woodside, N. Y.) were cast using phosphate-bonded investment with the lost wax technique. A commercial porcelain (Ceramco Opaqe, Ceramco Inc., Woodside, N. Y.) was fused to the end of the rods to form the specimen shown in Figure 1. Here the geometry will produce tensile failure. Roughening of the rods prior to applying the porcelain was done with a medium grit stone to a roughness of 33 uin. A surface roughness analyzer (Profilometer, Micrometrical Div., Bendix Corp., Ann Arbor, Mich.) was used to make the measurements. 


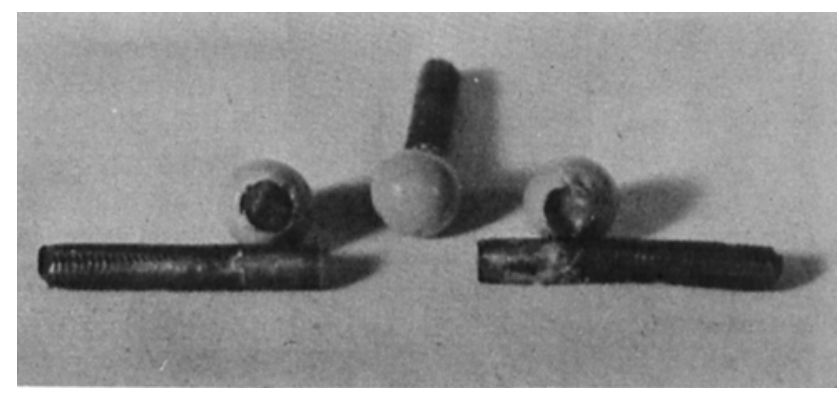

Fig. 1. Ceramco gold rod with porcelain baked on.

TABLE I

Factorial Design for Bond Strength Study

\begin{tabular}{lll}
\hline & Smooth & Rough \\
\hline \multirow{2}{*}{ Oxidized } & $\bar{x}=5420$ & $\bar{x}=5070$ \\
Sonoxidized & S.D. 160 & S.D. 100 \\
& $\bar{x}=3120$ & $\bar{x}=2850$ \\
& S.D. 160 & S.D. 240 \\
\hline
\end{tabular}

Oxidation was carried out by heating at $1800^{\circ} \mathrm{F}$ for $30 \mathrm{~min}$ prior to applying the porcelain.

The porcelain was applied by baking at 1850,1865 , and finally at $1875^{\circ} \mathrm{F}$. The porcelain was baked only on the end of the cylindrical casting and not on the shank. This was accomplished by means of a platinum matrix which is shown in Figure 2. Shearing stresses were minimized by this procedure. The large mass of porcelain in the region away from the edge of the gold helps to reduce stress concentrations and provides for a more even stress distribution.

The strength of the junction was determined by measuring the force necessary to detach the rod from the porcelain by means of a tensile-testing machine (Riele Testing Machine, East Moline, Illinois).

\section{Results}

The mean value and standard deviation for each of the four conditions are recorded in Table I. After each test, the gold surface was examined for residual porcelain. The values used in this study are 


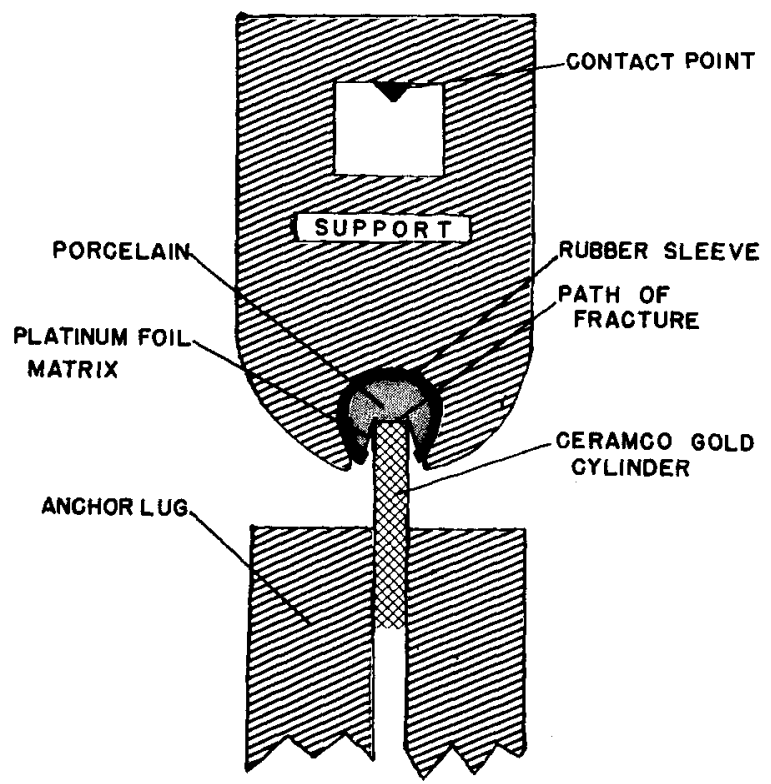

Fig. 2. Detail of specimen preparation shown in Fig. 1 .

only those which fractured at the interface. The significance of the results were tested by means of analysis of variance, as shown in Table II. At the $99 \%$ confidence level, the value obtained from statistical tables is 7.56. ${ }^{6}$ The calculated values of $F$ are shown in Table II and are much greater than 7.56. This indicates that the oxidized surface had a stronger bond than the nonoxidized surface, also the smooth surface resulted in a stronger bond than the grossrough surface. No interaction was found between the rough, smooth, and the oxidized-nonoxidized samples.

TABLE II

Analysis of Variance

\begin{tabular}{lrrrr}
\hline \multicolumn{1}{c}{ Sources } & $\begin{array}{c}\text { Sum } \\
\text { of squares }\end{array}$ & D.F. & $\begin{array}{c}\text { Mean } \\
\text { square }\end{array}$ & " $F "$ \\
\hline Rough and smooth (rows) & $\mathbf{7 5 6 4 . 5}$ & 1 & 7564.5 & 22.5 \\
Oxidized and nonoxidized (columns) & $407,253.12$ & 1 & $407,253.12$ & 1212.4 \\
Interaction & 136.12 & 1 & 136.12 & 0.40 \\
Experimental errors & 9404.25 & 28 & 335.87 & - \\
\hline
\end{tabular}




\section{Discussion}

The values obtained in this study for the porcelain-gold bond are lower than those obtained by other tests of similar nature. Shell and Nielson, using similar alloys and porcelain, report bond strengths of 9900 psi for roughened surfaces and 10220 psi for smooth surfaces. The lower values obtained in this experiment are due to the fact that only the tensile strengths of the specimens were measured.

The above data indicates that oxide formation is of primary importance in the strength of the bond. This oxide layer may act as a transition layer between the metallic and the ceramic phases, or it may create a microscopic surface roughness. Either case would

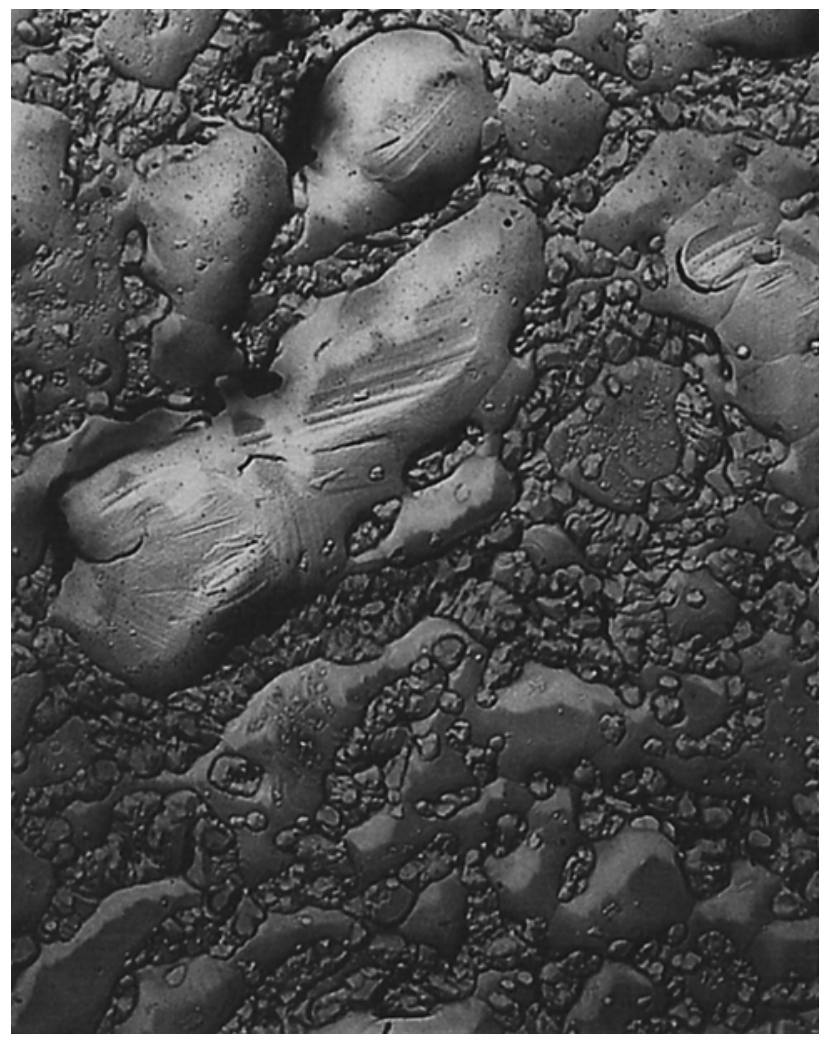

Fig. 3. Electron micrograph of chromium-shadowed replica of Ceramco surface after being oxidized at $1800^{\circ} \mathrm{F}$ for $30 \mathrm{~min}(7735 \times)$. 
result in strengthening the bond. The electron micrograph (Fig. 3) illustrates the nature of the polished gold surface after it has been oxidized at $1800^{\circ} \mathrm{F}$ for $30 \mathrm{~min}$. This shows microsurface roughness which is present on the surfaces of the oxidized specimens. The fact that the smooth surface specimens gave higher strengths than the rough specimens is not, easily explained, but indicates that surface roughening is an unnecessary practice. However, the effect of gross roughness is a second-order one in comparison to the influence of oxidation on bond strength.

\section{References}

1. J. S. Shell and J. P. Neilsen, J. Dent. Res., 41, 1424 (1962).

2. J. F. Johnston, R. W. Dykema, and D. M. Cunningham, J. Pros. Dent., 6, 811 (1956).

3. M. Silver, G. Klein, and M. C. Howard, J. Pros. Dent, 10, 1055 (1960).

4. F. Custer, J. Dent. Res., 40, 94 (1962).

5. R. C. Vickery, "Nature of attachment forces in ceramco-porcelain gold system," Microfilm Annual Meeting of the Dental Materials Group of the IADR, March, 1967.

6. A. J. Duncan, Quality Control and Industrial Statistics, Richard D. Irwin, Inc., Homewood, Ill., 1955, Table J, p. 618.

Received July 9, 1968 